\title{
Serological Update on Celiac Disease Diagnostics in Adults
}

\author{
Tsvetelina V. Velikova ${ }^{1, *}$, Zoya A. Spassova ${ }^{2}$, Kalina D. Tumangelova-Yuzeir ${ }^{3}$, Ekaterina K. Krasimirova ${ }^{3}$, \\ Ekaterina I. Ivanova-Todorova ${ }^{3}$, Dobroslav S. Kyurkchiev ${ }^{3}$, Iskra P. Altankova ${ }^{1}$ \\ ${ }^{1}$ Clinical Immunology, University Hospital “Lozenetz”, Sofia 1407, Bulgaria \\ ${ }^{2}$ Clinic of Gastroenterology, University Hospital "St. Ivan Rilski”, Medical University, Sofia 1431, Bulgaria \\ ${ }^{3}$ Laboratory of Clinical Immunology, University Hospital "St. Ivan Rilski”, Department of clinical laboratory and clinical immunology, \\ Medical University, Sofia 1431, Bulgaria \\ *Corresponding author: tsvelikova@medfac.mu-sofia.bg
}

\begin{abstract}
Celiac disease (CD) is an inflammatory disorder of the small intestines which serological diagnosis has come to the forefront with the development of the immunological testing. We aimed to explore the performance characteristics of a panel of serological tests in patients with $\mathrm{CD}$. We assessed the serum levels of anti-tissue transglutaminase (anti-tTG), anti-deamidated gliadin peptides (anti-DGP), anti-actin (AAA), anti-gliadin antibodies (AGA) and cytokine IL-17A by performing ELISA; and anti-tTG, AGA and anti-Saccharomyces cerevisiae antibodies (ASCA) by immunoblot in 35 newly diagnosed adult patients with biopsy-proven CD and 25 age- and sex-matched healthy persons. The average serum levels of anti-tTG, anti-DGP, AGA, AAA, and ASCA were at significantly higher levels in patients with $\mathrm{CD}$ compared to healthy persons $(\mathrm{p}<0.001)$. We also observed that the serum level of IL-17A was about 70 times higher in $\mathrm{CD}$ patients than in the healthy persons $(\mathrm{p}=0.027)$. Anti-DGP antibodies showed highest diagnostic sensitivity (100\%), followed by AGA and anti-tTG antibodies within the CD group. ROC curve analysis revealed the excellent performance of anti-DGP, anti-tTG, and AGA in the diagnosis of CD patients (AUC 1.000, 0.994, 0.992 respectively, $\mathrm{p}<0.001$ ). Although the diagnosis of CD relays on biopsy, immunological serological testing could be employed with advantages in diagnosis and monitoring of $\mathrm{CD}$ patients.
\end{abstract}

Keywords: celiac disease, autoantibodies, anti-tTG, anti-DGP, anti-F-actin antibodies, anti-gliadin antibodies, $I L-17 A$

Cite This Article: Tsvetelina V. Velikova, Zoya A. Spassova, Kalina D. Tumangelova-Yuzeir, Ekaterina K. Krasimirova, Ekaterina I. Ivanova-Todorova, Dobroslav S. Kyurkchiev, and Iskra P. Altankova, "Serological Update on Celiac Disease Diagnostics in Adults." International Journal of Celiac Disease, vol. 6, no. 1 (2018): 20-25. doi: 10.12691/ijcd-6-1-8.

\section{Introduction}

Celiac Disease (CD) is one of the most prevalent digestive conditions affecting at least $1 \%$ of the population worldwide, and evidence suggests that prevalence is increasing [1]. The diagnosis relies on strict criteria (clinical, histological, immunological, genetic) $[1,2,3]$. Since the variable presentation, including typical and atypical symptoms, CD can be difficult to diagnose, and the physicians need to possess a low threshold for CD testing for suspected and high-risk patients [1].

Biopsy testing, which is a gold standard for confirmation of $\mathrm{CD}$ diagnosis to this day, has many disadvantages: invasiveness, need for collecting $\geq 5$ duodenal biopsies with two samples from the duodenal bulb due to the patchy mucosal damage in up to $70 \%$ of patients; only $13 \%$ of patients demonstrated characteristic enteropathy located to duodenal bulb [1]. These reasons along with the accumulated knowledge of the $\mathrm{CD}$ pathogenesis have served as a catalyst for the development of serologic diagnostic tools [1]. Nowadays, the positive celiac-specific autoantibodies along with enteropathy are the most commonly used markers for gluten-related mucosal damage [4]. Moreover, all available guidelines for CD diagnosis include the combined use of biopsy and serologic tests. Serologic testing is performed first to identify patients who should undergo intestinal biopsy [1]. On the other hand, up to $3 \%$ of $C D$ patients may exert negative serologic results, or low and fluctuating titers, and for them, the upper endoscopy with biopsy is beneficial for diagnosis confirmation. Genetic testing is also available (HLA-DQ2.5 and HLADQ8 are useful for their acceptable negative predictive value) [5].

Currently, most celiac serology worldwide is performed with commercial ELISA kits. Starting with anti-gliadin (AGA) testing, which antibodies are produced in response to gliadin, a prolamin found in wheat, then passing by anti-endomysium (EMA) and anti-tissue transglutaminase (anti-tTG) examination, up to anti-deamidated gliadin peptides (anti-DGP) antibodies. DGPs are peptides formatted after the selective deamidation of gliadin by the action of intestinal tTG in the mucosa [1]. DGPs can bind with high affinity to MHC class II molecules DQ2 or DQ8 on antigen-presenting cells of $\mathrm{CD}$ patients with consecutive 
stimulation of inflammatory $\mathrm{T}$ cell-mediated response in the small intestines [1]. Furthermore, selective deamidation of gliadin results in a dramatic increase in test sensitivity of anti-DGP antibodies [6]. The recently described celiacrelated test is anti-F-actin antibodies (AAA), based on the presumption that villous atrophy also affects the actin - the primary component of cytoskeleton microfilaments, which is also a target for smooth muscle antibodies with the tubular pattern (ASMA-T) [7].

Highly accurate serologic assays allow not only noninvasive screening and detection but also have shown the growing prevalence of $\mathrm{CD}$ in many populations. Nevertheless, the CD is a benign disorder with a good prognosis in those patients that can adhere to a gluten-free diet (GFD) [2,5]. It is essential to monitor the GFD adherence with non-invasive reliable and cost-effective tests, such as serologic ones.

The most abundant commercially available serologic tests for $\mathrm{CD}$ are enzyme-linked immunosorbent assay (ELISA)-based, but there are also immunofluorescence and blotting methods. The growing number of available serologic CD tests needs a reevaluation of their diagnostic power and performance [8]. Thus, we aimed to compare the performance characteristics of a panel of serological tests in a Bulgarian cohort of adult patients with $\mathrm{CD}$, including auto-antibodies anti-tTG, anti-DGP, AGA, AAA, by ELISA and Line blot methods. We chose to assess the serum samples of the $\mathrm{CD}$ patients also for the cytokine IL-17A, as long as some recent studies documented ongoing cytokine interplay in CD mucosa [9], as well as the IL-17A was confirmed in the mucosal immune response when villous atrophy had developed [10].

\section{Material and Methods}

\subsection{Subjects}

Sixty persons were included in the study: 35 consecutive newly diagnosed patients with biopsy-proven $\mathrm{CD}$ and 25 age- and sex-matched healthy persons.

CD patients were recruited at the Clinic of gastroenterology at University Hospital St. Ivan Rilski, Sofia, and the diagnosis was based on the set of anamnestic, clinical, laboratory and instrumental studies of the patients. For all patients, Marshal score was assessed by the number of intraepithelial lymphocytes (IEL/100), enterocytes, villous atrophy, crypt hyperplasia: from Marsh I to Marsch IIIc. The mean age of the patients was $41 \pm 3$ (20-65) years; four were men $(11.4 \%)$ and thirty-one - women $(88.6 \%)$. The exclusion criteria for patients were the following but not limited to: proved infectious diarrhea, NSAIDs usage, melena, other systemic severe or psychiatric illness.

The control group of healthy persons comprises of six men $(24 \%)$ and nineteen women $(76 \%)$ at mean age $29 \pm 5$ years. Gastrointestinal diseases, systemic severe or psychiatric illnesses were excluded for these subjects.

All subjects of the study were informed about the purpose of the experiment, and a written informed consent was obtained from all participants. The study was approved by the Ethic Committee of the Medical University of Sofia, and the research was performed according to the local hospitals`ethical considerations.

\subsection{Methods}

We examined the serum levels of patients and healthy controls for several celiac-related autoantibodies by ELISA and line blot, IgA and the cytokine IL-17A. All immunological testing was performed at the Laboratory of Clinical Immunology, University Hospital "St. Ivan Rilski”, Sofia, according to manufacturer's instructions.

\subsubsection{Enzyme Immunoassays}

- Anti-tissue transglutaminase (anti-tTG) antibodies (Anti-Tissue Transglutaminase Screen IgA+IgG, Orgentec Diagnostika $\mathrm{GmbH}$ );

- Anti-deamidated gliadin peptides (anti-DGP) antibodies (Quanta Lite Celiac DGP Screen IgA+IgG, Inova Diagnostics, Inc., San Diego, USA);

- Anti-actin (AAA) antibodies (Quanta Lite F-Actin IgA ELISA, Inova Diagnostics, Inc., San Diego, USA);

- Anti-gliadin (AGA) antibodies (Anti-Gliadin Screen IgA+IgG, Orgentec Diagnostika GmbH);

- Interleukin 17A (Human IL-17A ELISA kit, Diaclone, GenProbe, France);

\subsubsection{Line Immunoblot}

- Anti-tTG (IgG), AGA (IgG), and anti-Saccharomyces cerevisiae antibodies (ASCA) (IgG) (Line blot testing - Seraline Line Immunoassay - Seraline Zoliakie - 3 IgG, Seramun Diagnostica $\mathrm{GmbH}$ ). The assay strips were scanned and evaluated with IvD-registered Seraline Scan software with hardware key (Seramun Diagnostica GmbH).

\subsubsection{Nephelometry}

- IgA - MININEPHTM HUMAN IgA KIT (The Binding Site Group, UK), performed on a laser nephelometer Minineph plus.

\subsubsection{Statistical Methods}

We analyze the raw data with the software package for statistical analysis (SPSS $\AA$, IBM 2009), v. 19. The results were accepted for significant if $p<0.05$. Diagnostic specificity, sensitivity, positive and negative predictive value were calculated by adapted formulas. The diagnostic accuracy for each test was calculated by using the manufacturers ' recommended cut-off values. We accepted the results above these cut-offs, including the dubious ones, as positive results.

\section{Results}

\subsection{Serum Levels of Celiac-related Autoantibodies and IL-17A}

The average serum levels of anti-tTG, anti-DGP, AGA and AAA were significantly higher in patients with $C D$ compared to healthy persons, independently of the method used. The levels of autoantibodies in serum samples of the healthy persons remained below the cut-off values of the 
tests $(\mathrm{p}<0.001) \quad$ (Figure 1A). Five out of thirty-five patients $(14.3 \%)$ were positive for anti-DGP and negative for anti-tTG antibodies. We also observed that the serum level of IL-17A was about 70 times higher in CD patients than in the healthy persons $(p=0.027)$ (Table 1$)$. We found ASCA above the cut-off value in about $1 / 3$ of the CD

A

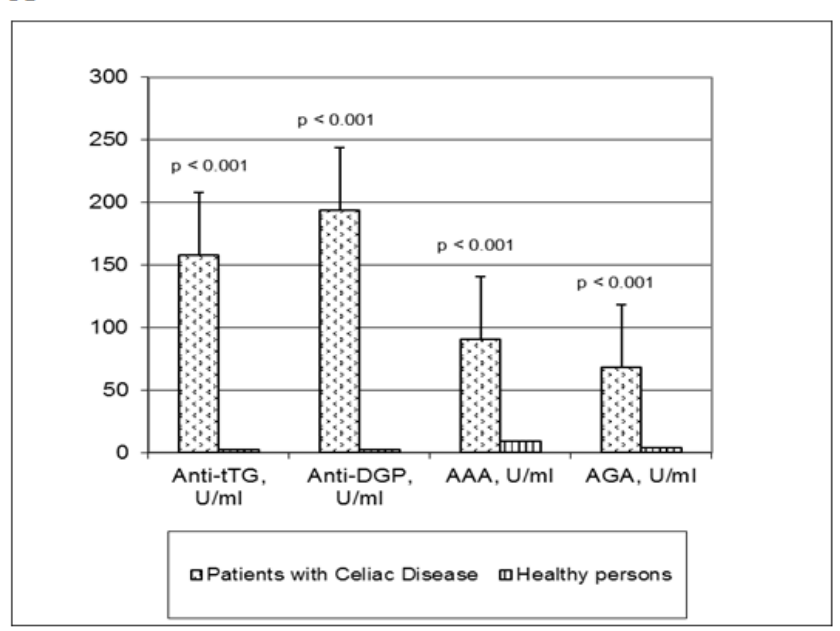

patients. Nevertheless, the average level in CD patients was higher than in the healthy control group $(p=0.002)$ (Figure 1B). The average level of serum $\operatorname{IgA}$ was $3.2 \pm 1.3 \mathrm{~g} / \mathrm{l}$, within the reference ranges of $0.71-3.60 \mathrm{~g} / \mathrm{l}$. No one of the recruited $\mathrm{CD}$ patients showed selective IgA deficiency.

B



Figure 1. Serum level of the autoantibodies, investigated by ELISA (A) or line blot (B), within the study groups

Table 1. Serum Level of the Autoantibodies and IL-17A, Investigated by ELISA or Line blot, within the Study Groups. Results are Presented as Mean \pm SE (Range). Line blot Measurements are Relative Value of Intensity.

\begin{tabular}{|c|c|c|c|c|}
\hline & Parameter & Celiac disease patients & Healthy individuals & $P$ value \\
\hline \multirow{5}{*}{ ELISA } & Anti-tTG, U/ml & $157.46 \pm 12.63$ & $2.14 \pm 0.30$ & $<0.001$ \\
\hline & Anti-DGP, U/ml & $193.38 \pm 29.21$ & $2.50 \pm 0.65$ & $<0.001$ \\
\hline & AGA, $\mathrm{U} / \mathrm{ml}$ & $68.35 \pm 11.80$ & $3.85 \pm 0.79$ & $<0.001$ \\
\hline & $\mathrm{AAA}, \mathrm{U} / \mathrm{ml}$ & $90.33 \pm 10.74$ & $9.02 \pm 0.84$ & $<0.001$ \\
\hline & IL-17A, pg/ml & $65.49 \pm 36.78$ & $0.09 \pm 0.06$ & 0.027 \\
\hline \multirow{3}{*}{ Line blot } & Anti-tTG & $3.54 \pm 0.41$ & $0.08 \pm 0.02$ & $<0.001$ \\
\hline & AGA & $2.97 \pm 0.41$ & $0.25 \pm 0.07$ & $<0.001$ \\
\hline & ASCA & $0.64 \pm 0.22$ & $0.04 \pm 0.02$ & 0.002 \\
\hline
\end{tabular}

Table 2. Serum level of the autoantibodies and IL-17A, investigated by ELISA or line blot, within the

\begin{tabular}{|c|c|c|c|c|c|c|}
\hline & Anti-tTG (ELISA) & $\begin{array}{c}\text { Anti-tTG } \\
\text { (Line blot) }\end{array}$ & $\begin{array}{c}\text { AGA } \\
\text { (ELISA) }\end{array}$ & $\begin{array}{c}\text { AGA } \\
\text { (Line blot) }\end{array}$ & $\begin{array}{c}\text { Anti-DGP } \\
\text { (ELISA) }\end{array}$ & $\begin{array}{c}\text { AAA } \\
\text { (ELISA) }\end{array}$ \\
\hline Sensitivity & $85.7 \%$ & $80 \%$ & $90 \%$ & $68.6 \%$ & $100 \%$ & $77 \%$ \\
\hline Specificity* & $100 \%$ & $100 \%$ & $100 \%$ & $94 \%$ & $97 \%$ & $100 \%$ \\
\hline PPV & $85.7 \%$ & $80 \%$ & $90 \%$ & $92 \%$ & $97.2 \%$ & $77 \%$ \\
\hline NPV & $83.3 \%$ & $78.1 \%$ & $96 \%$ & $52 \%$ & $100 \%$ & $75.6 \%$ \\
\hline
\end{tabular}

\subsection{Performance Characteristics of Celiac-related Autoantibodies}

Anti-DGP antibodies, assessed by ELISA, showed the best diagnostic sensitivity (100\%), followed by AGA, anti-tTG (ELISA), anti-tTG (Line blot), AAA (ELISA) and AGA (Line blot) within the CD group (Table 2). Specificity, calculated according to the ability of the test to distinguish healthy persons from patients with $\mathrm{CD}$, showed $100 \%$ for anti-tTG (ELISA and Line blot), and AAA (ELISA) and acceptable percentage for AGA (Line blot) and anti-DGP (ELISA). The best PPV and NPV were obtained for anti-DGP (ELISA), followed by AGA
(ELISA and Line blot) and anti-tTG (ELISA and Line blot) (Table 2). AAA showed the least satisfied PPV and NPV.

ROC curve analysis revealed the excellent performance of anti-DGP in diagnosis of CD patients with the highest area under the curve and the best specificity (AUC 1.000, $\mathrm{p}<0.001$ ), followed by anti-tTG, AGA, and AAA, assessed by ELISA, (AUC 0.994, 0.992, 0.878, respectively, $\mathrm{p}<0.001$ ) (Figure 2A). Anti-tTG, AGA, and ASCA, assessed by Line blot, also showed very good performance (AUC 0.994, 0.893, 0.759, respectively, $\mathrm{p}<0.001$ ) (Figure 2B). Overall the diagnostic accuracy of anti-DGP, anti-tTG, AGA, and AAA, assessed by ELISA and/or Line blot, was comparable. 
A

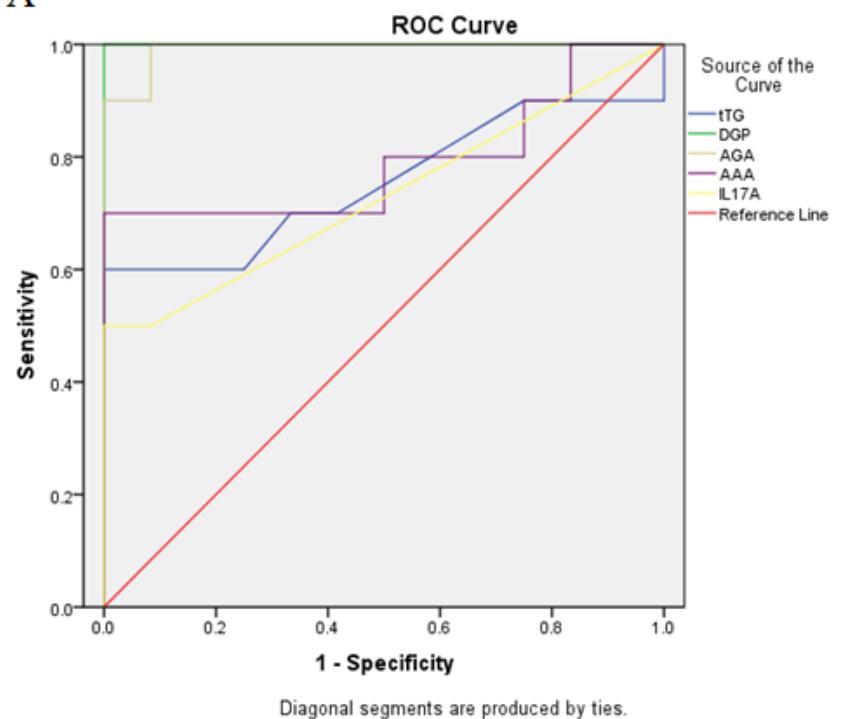

B

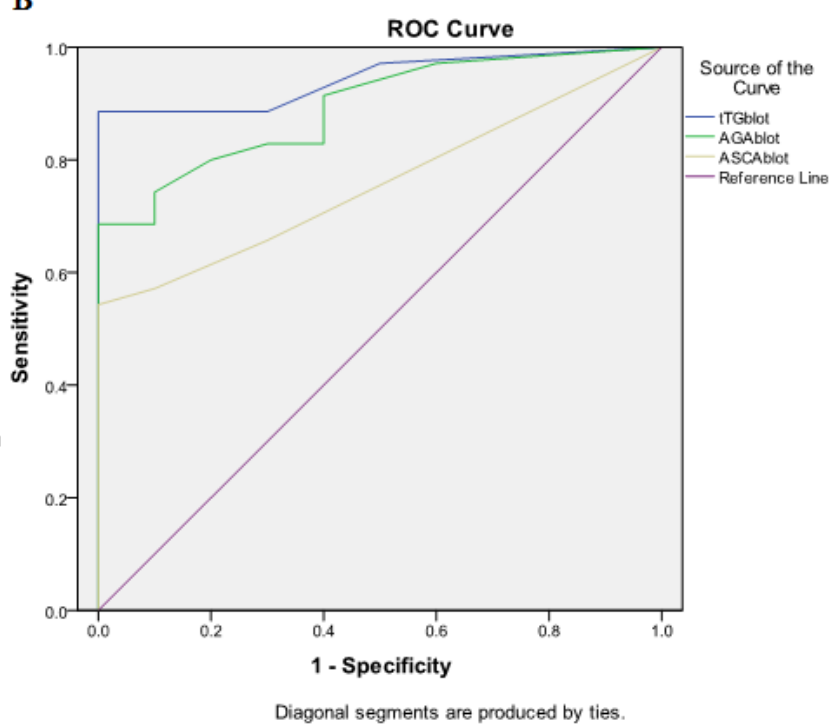

Figure 2. Receiver Operating Characteristic (ROC) curve analyses of the autoantibodies and IL-17A, assessed by ELISA (A) and lineblot (B)

Table 3. Receiver Operating Characteristic (ROC) Curve Analyses of the Autoantibodies and IL-17A, Assessed by ELISA and Line blot

\begin{tabular}{|c|c|c|c|c|}
\hline & Parameter & Area under the curve (AUC) & $95 \% \mathrm{CI}$ & $P$ value \\
\hline \multirow{5}{*}{ ELISA } & Anti-tTG & 0.919 & $0.842-0.997$ & $<0.001$ \\
\hline & Anti-DGP & 1.000 & $1.000-1.000$ & $<0.001$ \\
\hline & AGA & 0.992 & $0.965-1.000$ & 0.014 \\
\hline & AAA & 0.878 & $0.784-0.971$ & $<0.001$ \\
\hline & IL-17A & 0.628 & $0.487-0.769$ & 0.095 \\
\hline \multirow{3}{*}{ Line blot } & Anti-tTG & 0.944 & $0.885-1.000$ & $<0.001$ \\
\hline & AGA & 0.893 & $0.811-0.974$ & $<0.001$ \\
\hline & ASCA & 0.759 & $0.635-0.883$ & 0.002 \\
\hline
\end{tabular}

IL-17A showed non-significant AUC (Table 3).

\subsection{Correlation between Tests}

We found significant correlations between tests (Table 4). The moderate to strong correlations were observed for the following pairs: anti-tTG (ELISA) - anti-tTG (Line blot) $(\mathrm{r}=0.805, \mathrm{p}<0.001)$, anti-tTG (ELISA) - AAA (ELISA) $(\mathrm{r}=0.663, \mathrm{p}<0.001)$ and anti-DGP (ELISA) - anti-tTG (Line blot) $(\mathrm{r}=0.672, \mathrm{p}=0.001)$. IL-17A did not show correlations with any of the tested autoantibodies (Table 4). We did not find associations between AAA and Marsch score of the patients.

Table 4. Correlation Between Tests` Results in Patients with Celiac Disease, the Data are Presented as Pearson or Spearman Coefficient, $r$, and Significance, $\mathbf{p}$.

\begin{tabular}{|c|c|c|c|c|c|c|c|c|}
\hline & $\begin{array}{l}\text { Anti-tTG } \\
\text { (ELISA) }\end{array}$ & $\begin{array}{c}\text { Anti-DGP } \\
\text { (ELISA) }\end{array}$ & $\begin{array}{c}\text { AGA } \\
\text { (ELISA) } \\
\end{array}$ & $\begin{array}{c}\text { AAA } \\
\text { (ELISA) }\end{array}$ & $\begin{array}{l}\text { IL-17A } \\
\text { (ELISA) }\end{array}$ & $\begin{array}{c}\text { Anti-tTG } \\
\text { (Line blot) }\end{array}$ & $\begin{array}{c}\text { AGA } \\
\text { (Line blot) }\end{array}$ & $\begin{array}{c}\text { ASCA } \\
\text { (Line blot) }\end{array}$ \\
\hline Anti-tTG (ELISA) & & $\begin{array}{l}\mathrm{r}=0.363 \\
\mathrm{p}=0.126\end{array}$ & $\begin{array}{l}\mathrm{r}=0.268 \\
\mathrm{p}=0.455\end{array}$ & $\begin{array}{l}\mathrm{r}=0.663 \\
\mathrm{p}<0.001\end{array}$ & $\begin{array}{l}\mathrm{r}=0.305 \\
\mathrm{p}=0.085\end{array}$ & $\begin{array}{l}\mathrm{r}=0.805 \\
\mathrm{p}<0.001\end{array}$ & $\begin{array}{l}\mathrm{r}=0.561 \\
\mathrm{p}<0.001\end{array}$ & $\begin{array}{l}\mathrm{r}=0.474 \\
\mathrm{p}<0.001\end{array}$ \\
\hline anti-DGP (ELISA) & & & $\begin{array}{l}\mathrm{r}=-0.065 \\
\mathrm{p}=0.903\end{array}$ & $\begin{array}{l}\mathrm{r}=0.361 \\
\mathrm{p}=0.033\end{array}$ & $\begin{array}{l}\mathrm{r}=0.160 \\
\mathrm{p}=0.500\end{array}$ & $\begin{array}{l}\mathrm{r}=0.672 \\
\mathrm{p}=0.001\end{array}$ & $\begin{array}{l}\mathrm{r}=0.339 \\
\mathrm{p}=0.144\end{array}$ & $\begin{array}{l}\mathrm{r}=-0.033 \\
\mathrm{p}=0.890\end{array}$ \\
\hline AGA (ELISA) & & & & $\begin{array}{l}\mathrm{r}=0.051 \\
\mathrm{p}=0.890\end{array}$ & $\begin{array}{l}\mathrm{r}=-0.191 \\
\mathrm{p}=0.597\end{array}$ & $\begin{array}{l}\mathrm{r}=0.539 \\
\mathrm{p}=0.108\end{array}$ & $\begin{array}{l}\mathrm{r}=0.355 \\
\mathrm{p}=0.314\end{array}$ & $\begin{array}{l}\mathrm{r}=0.308 \\
\mathrm{p}=0.389\end{array}$ \\
\hline AAA (ELISA) & & & & & $\begin{array}{l}\mathrm{r}=-0.006 \\
\mathrm{p}=0.972\end{array}$ & $\begin{array}{l}\mathrm{r}=0.311 \\
\mathrm{p}=0.069\end{array}$ & $\begin{array}{l}\mathrm{r}=0.193 \\
\mathrm{p}=0.267\end{array}$ & $\begin{array}{l}\mathrm{r}=0.054 \\
\mathrm{p}=0.759\end{array}$ \\
\hline IL-17A (ELISA) & & & & & & $\begin{array}{l}\mathrm{r}=0.024 \\
\mathrm{p}=0.891\end{array}$ & $\begin{array}{l}\mathrm{r}=0.304 \\
\mathrm{p}=0.075\end{array}$ & $\begin{array}{l}\mathrm{r}=-0.002 \\
\mathrm{p}=0.989\end{array}$ \\
\hline Anti-tTG (Line blot) & & & & & & & $\begin{array}{l}\mathrm{r}=0.539 \\
\mathrm{p}=0.108\end{array}$ & $\begin{array}{l}\mathrm{r}=0.406 \\
\mathrm{p}=0.016\end{array}$ \\
\hline AGA (Line blot) & & & & & & & & $\begin{array}{c}\mathrm{r}=0.18 \\
\mathrm{p}=0.919\end{array}$ \\
\hline ASCA (Line blot) & & & & & & & & \\
\hline
\end{tabular}




\section{Discussion}

In the present study, we evaluated five commercial immune assays (four ELISAs and one Line blot) for detection of celiac-related autoantibodies along with IL-17A in a Bulgarian cohort of 35 consecutive newly diagnosed and biopsy-proven CD patients and 25 healthy controls. We also aimed to compare their diagnostic accuracy for the $\mathrm{CD}$ diagnosis.

As long as some CD patients with a selective IgA deficiency will test false-negative for $\operatorname{IgA}$ antibodies $[1,6]$, we chose tests with $\operatorname{IgG}+\operatorname{IgA}$ antibodies specificity to avoid this severe drawback of the antibody detection. However, no one of the tested subjects possessed selective IgA deficiency.

\subsection{AGA}

In our study, the performance characteristics of AGA were acceptable regardless of the method used - with sensitivity (68.6-80\%), specificity (94-100\%), PPV (90$92 \%)$ and NPV (52-96\%). These results are in accordance with the results of the meta-analysis of Leffler et al., except for the PPV. We observed much higher PPV than the other studies (18-31\%) [8]. According to some experts, AGA testing should not be considered a part of routine CD testing anymore due to its high false positives results in patients with other gastrointestinal diseases (i.e., atrophic gastritis) [8]. AGA testing had already fallen out of favor in the last century due to its low PPV which is shown to be less than $30 \%$ in the general population and when more accurate testing, such as EMA, became widely available [1]. Despite the well-documented high diagnostic accuracy of EMA, it has rather more disadvantages: high cost, subjectiveness, laborintensiveness, requiring experienced personnel to perform an individual reading of each sample on a microscope, ethic issues regarding using of either monkey esophagus or human umbilical cord tissue as a substrate $[8,11]$. On this background, we chose not to test our patients for EMA. Especially when the antigen of EMA has already been identified as tTG; there are enough commercially available ELISA detecting anti-tTG [11], and having in mind the comparable sensitivity and specificity of both tests [12].

\subsection{Anti-tTG Antibodies}

Referring anti-tTG testing, we observed worse performance than the AGA testing (Table 4). Nevertheless, the sensitivity of anti-tTG $(\operatorname{IgA}+\operatorname{IgG})$ varied between $80-85.7 \%$ (ELISA and Line blot) and specificity $100 \%$ at the cut-off recommended by the manufacturer. Other studies announced similar data (sensitivity of IgA anti-tTG 78-100\% and IgG anti-tTG $45-95 \%$, and specificity of IgA anti-tTG 90-100\% and IgG anti-tTG 94-100\%) [8,13]. PPV and NPV for anti-tTG testing in our study were also satisfied $80-85.7 \%$ and $78.1-83.3 \%$, respectively, like other studies $-42-72 \%$ and $99 \%$, respectively) [8]. These results made anti-tTG, particularly of IgA class, the best serologic assay for CD [6].

\subsection{Anti-DGP Antibodies}

After Schwertz et al. showed in 2004 that the detection of anti-DGP antibodies could be a valuable tool for the diagnosis of CD [14], many studies confirmed that the performance of IgG anti-DGP antibodies is comparable to the performance of IgA anti-tT [6]. The median sensitivity of anti-DGP testing for CD was reported 0.91 (0.69-0.984) and the median specificity - $0.969(0.903-0.98)$ like the median PPV 0.945 (0.892-0.989) and NPV- 0.972 (0.852-0.989) [13]. Here, we obtained similar results: $100 \%, 97 \%, 97.2 \%$ and $100 \%$, respectively. The combined IgA+IgG anti-DGP testing, which we also used in our study, was reported with the following results: $97 \%$ (75-99), 95\% (87-100\%), 51\% and 99\%, respectively [8]. Combining two or more Ig class, especially DGP (IgA and $\operatorname{IgG}$ ) and tTG (IgA and IgG) as in our study, was shown as preferable as compared to the single class as concluded by others [13]. Furthermore, all combined assays, such as anti-DGP/tTG screen, had higher sensitivities than every single assay alone. These observations encouraged many clinicians to diagnose $\mathrm{CD}$ accurately in different clinical cases and to avoid biopsy in a high proportion of subjects [4].

\subsection{AAA}

The desirable non-invasive assessing of any villous atrophy during follow-up of the $\mathrm{CD}$ patients was close with the introduction of AAA as a serum marker for mild to severe villous atrophy, and also for monitoring the disease [7,15]. However, we did not find associations between AAA levels and Marsch score of our patients. This may be due to relatively small sample size, and heterogeneity in Marshal score obtained in our patients. Another explanation is that up to $69 \%$ of the CD patients exert high levels of AAA [15], whereas only $32 \%$ of our patients were positive for AAA.

\subsection{ASCA}

Here, we tested CD patients for the ASCA while this antibody was included in the used Line blot. However, we found that $30 \%$ of our patients were positive for ASCA, and their levels were evaluated as significantly higher than the healthy control group. Granito et al. found $59 \%$ of CD patients to be ASCA positive, but without correlations with severity of small intestinal mucosal damage [16]. Furthermore, they observed that in asymptomatic patients, ASCA positivity was predictive of the disease, as well as associated with "potential/silent" CD [16].

\subsection{The Role of Autoantibodies in Clinical Practice}

Some authors have proposed that histology testing could no longer be obligatory for the CD diagnosis based on the very high PPVs of reliable serological tests [4]. In view of our excellent results for PPV and NPV of autoantibodies tested, we could incline our conclusions in that direction. However, other authors reminded that PPV and NPV could vary widely across populations [8], as well as they depend strongly on the prevalence of the disease [17].

Patients with CD need a permanent GFD after diagnosing, and they should be monitored periodically and routinely tested for anti-tTG antibodies to evaluate the compliance with the diet [2]. For the follow-up, however, anti-DGP antibodies have been shown to be more sensitive in unveiling 
compliance to GFD compared to anti-tTG, even though the enhanced diagnostic sensitivity of the latter [18]. However, in clinical practice, even strongly positive anti-tTG IgA results did not show necessarily correlation with the severity of the villous atrophy, thus, they are unlikely to be useful for monitoring diet adherence [17]. It is important to note that all antibody-based serologic tests are expected to normalize on a GFD, so test accuracy depends on ongoing gluten consumption [8]. These findings regard further investigations.

We should also point out that individual CD patients have negative celiac serologies, inclusive some of our patients, which was negative for one or more celiac-related antibodies. The major possible explanations for these CD patients with villous atrophy but without positive autoantibodies, are: GFD administration at the time of testing, IgA deficiency, non-celiac enteropathy or merely seronegative $\mathrm{CD}[1]$.

\subsection{IL-17A}

Gluten-specific IL-17A-producing CD4+ and CD4+CD8+ cells have been recently observed in the duodenum of $\mathrm{CD}$ patients $[19,20]$, as well as upregulated IL-17A mRNA in active CD [10]. However, DGP-specific T-cells have not been shown to secrete IL-17A, which suggests that IL-17 production is realized at a later stage of mucosal inflammation [10]. We observed significantly higher serum levels of IL-17A in CD patients compared to healthy controls $(65.49 \mathrm{pg} / \mathrm{ml}$ vs. $0.09 \mathrm{pg} / \mathrm{ml}, \mathrm{p}=0.027)$. In line with this are the observations of other authors detected CD4+IL-17+cells in peripheral blood by flow cytometry [9]. However, we ought to have in mind that serum levels do not necessarily mirror the mucosal levels. Moreover, the role of IL-17 in CD pathogenesis remains elusive. Thus, further studies should be performed to specify the potential of Th17 cells for therapeutic interventions, as well as the IL17 as a marker for active $\mathrm{CD}$ or villous atrophy.

\section{Conclusion}

Although the diagnosis of CD relays on the gold standard - biopsy, serological testing could be employed with advantages in diagnosis and monitoring of CD patients. The improved multiple useful modalities, including serologic measurements with high sensitivity and specificity, and growing awareness among physicians may help diagnosing more accurately and more patients with $\mathrm{CD}$, regardless of the atypical manifestations and possible differential diagnosis. The role of IL-17A in CD pathogenesis is still debated and open to investigation.

\section{Acknowledgements}

This study was supported by Grant funding №12D/2013-2014, Project № 3-D, from the Medical University, Sofia.

\section{Conflict of Interests}

The authors have no competing interests.

\section{References}

[1] Kaswala, D.H., Veeraraghavan, G., Kelly, C.P., Leffler, D.A., "Celiac Disease: Diagnostic Standards and Dilemmas," Diseases, 3. 86-101. June 2015.

[2] Dias, J.A., "Celiac Disease: What Do We Know in 2017?," GE Port J Gastroenterol, 24. 275-278. November 2017.

[3] Arslan, N., "Towards Finding More Diagnostic Serological Markers in Celiac Disease: Can Deamidated Gliadin Peptide Antibodies Help to Our Babies?," International Journal of Celiac Disease, 1 (1). 27-28. 2013.

[4] Sugai, E., Moreno, M.L., Hwang, H.J., Cabanne, A., Crivelli, A., Nachman, F., Vázquez, H., Niveloni, S., Argonz, J., Mazure, R., La Motta, G., Caniggia, M.E., Smecuol, E., Chopita, N., Gómez, J.C., Mauriño, E., Bai, J.C., "Celiac disease serology in patients with different pretest probabilities: Is biopsy avoidable?," World $J$ Gastroenterol, 16 (25). 3144-3152. July 2010.

[5] Shannahan, S., Leffler, D.A., "Diagnosis and Updates in Celiac Disease," Gastrointest Endoscopy Clin N Am, 27 (2017). 79-92. January 2017.

[6] Vermeersch, P., Geboes, K., Mariën, G., Hoffman, I., Hiele, M., Bossuyt. X., "Diagnostic performance of IgG anti-deamidated gliadin peptide antibody assays is comparable to IgA anti-tTG in celiac disease," Clinica Chimica Acta, 411 (2010). 931-935. February 2010.

[7] Granito, A., Muratori, P., Cassani, F., et al., "Anti-actin IgA antibodies in severe coeliac disease," Clin Exp Immunol, 137 (2). 386-392. August 2004.

[8] Leffler, D.A., Schuppan, D., "Update on Serologic Testing in Celiac Disease," Am J Gastroenterol, 105. 2520-2524. December 2010.

[9] Ortega, C., Fernandez, S., Estevez, O.A., Aguado, R., Molina, I.J., Santamaria, M., "IL-17 Producing T Cells in Celiac Disease: Angels or Devils?," International Reviews of Immunology, 32. 534-543. September 2013.

[10] Cicerone, C., Nenna, R., Pontone, S., "Th17, intestinal microbiota and the abnormal immune response in the pathogenesis of celiac disease," Gastroenterol Hepatol Bed Bench, 8 (2). 117-122. March 2015.

[11] Dieterich, W., Ehnis, T., Bauer, M., Donner, P., Volta, U., Riecken, E.O., Schuppan, D., "Identification of tissue transglutaminase as the autoantigen of celiac disease," Nat. Med. 3 797-801. July 1997.

[12] Giersiepen, K., Lelgemann, M., Stuhldreher, N., Ronfani, L., Husby, S., Koletzko, S., Korponay-Szaby, I.R., ESPGHAN Working Group on Coeliac Disease Diagnosis, "Accuracy of diagnostic antibody tests for coeliac disease in children: summary of an evidence report," J Pediatr Gastroenterol Nutr 54. 229-241. February 2012.

[13] Schyum, A.C., Rumessen, J.J., "Serological testing for celiac disease in adults," United European Gastroenterology Journal, 1 (5). 319-325. October 2013.

[14] Schwertz, E., Kahlenberg, F., Sack, U., et al., "Serologic assay based on gliadin-related nonapeptides as a highly sensitive and specific diagnostic aid in celiac disease," Clin Chem, 50. 2370-5. October 2004

[15] Carrocio, A., Brusca, I., Iacono, G., et al., "IgA anti-actin antibodies ELISA in coeliac disease: a multicenter study," Dig Liver Dis, 39 (9). 818-823. September 2007.

[16] Granito, A., Zauli, D., Muratori, P., et al., “Anti - Saccharomyces cerevisiae and perinuclear anti - neutrophil cytoplasmic antibodies in coeliac disease before and after gluten free diet," Aliment Pharmacol Ther 2. 1881-887. April 2005.

[17] Lerner, A., Jeremias, P., Matthias, T., "Outside of Normal Limits: False Positive/Negative Anti TG2 Autoantibodies," International Journal of Celiac Disease, 3 (3). 87-90. July 2015.

[18] Monzani, A., Rapa, A., Fonio, A., Tognato, E., Panigati, L., Oderda, G., "Use of deamidated gliadin peptide antibodies to monitor diet compliance in childhood celiac disease," $J$ pediatr gastroeneterol nutr, 53 (1). 55-60. July 2011.

[19] Mazzarella, G., "Effector and suppressor T cells in celiac disease," World J Gastroenterol, 21 (24). 7349-7356. June 2015.

[20] Monteleone, I., Sarra, M., Del Vecchio Blanco, G., Paoluzi, O.A., Franze, E., Fina, D., Fabrizi, A., MacDonald, T.T., Pallone, F., Monteleone, G., "Characterization of IL-17A-Producing Cells in Celiac Disease Mucosa," J Immunol, 184. 2211-2218. February 2015 . 